\title{
Dosimetric impact of post-operative seroma reduction during radiotherapy after breast-conserving surgery
}

Tanja Alderliesten, $\mathrm{PhD}^{1}$, Suzanne den Hollander, RT(T) ${ }^{1}$, Tzu-I Jonathan Yang, BSRT(T) $)^{1,2}$, Paula H M Elkhuizen, $\mathrm{MD}, \mathrm{PhD}^{1}$, Anke $\mathrm{M}$ van Mourik, $\mathrm{PhD}^{1}$, Coen Hurkmans, $\mathrm{PhD}^{3}$, Peter Remeijer, $\mathrm{PhD}^{1}$, Corine van Vliet-Vroegindeweij, $\mathrm{PhD}^{1}$

${ }^{1}$ Department of Radiation Oncology, The Netherlands Cancer Institute - Antoni van Leeuwenhoek Hospital, Amsterdam, The Netherlands

${ }^{2}$ Yale University School of Medicine, New Haven, CT, U.S.A.

${ }^{3}$ Department of Radiation Therapy, Catharina Hospital, Eindhoven, The Netherlands

Corresponding Author: Corine van Vliet-Vroegindeweij, PhD, The Netherlands Cancer Institute-Antoni van Leeuwenhoek Hospital, Department of Radiation Oncology, Plesmanlaan 121, 1066 CX Amsterdam, The Netherlands, Phone: +31 20512 2243, Fax: +31 20669 1101, Email: c.v.vliet@nki.nl

Key words: Breast cancer, Adaptive image guided radiotherapy, Boost volume, Post-operative seroma

\begin{abstract}
Purpose: Three boost radiotherapy (RT) techniques were compared to evaluate the dosimetric effect of seroma reduction during RT after breast-conserving surgery (BCS).

Materials and methods: Twenty-one patients who developed seroma after BCS were included. Each patient underwent three CT scans: one week before RT $\left(\mathrm{CT}_{-1}\right)$, in the third $\left(\mathrm{CT}_{3}\right)$ and fifth $\left(\mathrm{CT}_{5}\right)$ week of RT. For each patient, three plans were generated. 1) SEQ: whole breast irradiation planned on $\mathrm{CT}_{-1}$, sequential boost planned on $\mathrm{CT}_{5}$, 2) SIB: simultaneous integrated boost planned on $\left.\mathrm{CT}_{-1}, 3\right)$ SIB adaptive radiation therapy (SIB-ART): planned on $\mathrm{CT}_{-1}$ and re-planned on $\mathrm{CT}_{3}$. Irradiated volumes, mean lung (MLD) and maximum heart dose $\left(\mathrm{HD}_{\max }\right)$ were projected and compared on $\mathrm{CT}_{5}$.

Results: On average 62\% seroma reduction during RT was observed. Volumes receiving $\geq 107 \%$ of prescribed whole breast dose were significantly smaller with SIB-ART compared to SEQ and SIB. The undesired volume receiving $\geq 95 \%$ of prescribed total dose was also significantly smaller with SIB-ART. For SEQ, SIB-ART and SIB respectively, small but significant differences were found in MLD (4.2 vs. 4.6 vs. $4.7 \mathrm{~Gy}$ ) and in $\mathrm{HD}_{\max }$ for patients with left-sided breast cancer (39.9 vs. 35.8 vs. $36.9 \mathrm{~Gy}$ ).

Conclusions: This study demonstrates a dosimetric advantage for patients with seroma when simultaneous integrated boost is used with re-planning halfway through treatment.
\end{abstract}

\section{Introduction}

Radiotherapy (RT) following breast-conserving surgery (BCS) has been proven to be effective in improving local control and long-term survival [1]. Following whole breast irradiation (WBI), an additional boost to the tumor bed was found to further decrease local recurrence [2]. In case the boost target volume $\left(\mathrm{TV}_{\text {boost }}\right)$ on the initial planning $\mathrm{CT}$ includes post-operative seroma, it is frequently assumed that it does not change significantly during RT. However, prior studies have demonstrated 36\% to 50\% seroma reduction prior to RT [3,4], and $22 \%$ to $62 \%$ during RT [5-9]. Due to seroma reduction, the volume of breast tissue not intended to receive boost RT will become excessive towards the end of treatment, with increasing risk of fibrosis and additional worsening of cosmetic outcome [10,11].

Though we now know that $\mathrm{TV}_{\text {boost }}$ is dynamic during $\mathrm{RT}$ and the irradiated volume should be kept as small as possible to optimize cosmetic results, no investigation has addressed the dosimetric consequences of different planning techniques specifically for boost volumes including seroma. Boost irradiation can be planned and delivered separately from or integrated with WBI. Planning of boost irradiation after WBI has the advantage of obtaining a second CT scan specifically for boost planning prior to boost irradiation, thereby accounting for seroma reduction during WBI. A large seroma reduction is primarily to be expected in patients with a large initial seroma volume [8,9], suggesting that a sequential boost will minimize irradiation overdose in this patient group. On the other hand, simultaneous integrated boost $[12,13]$, with or without re-planning halfway through RT, can provide the benefit of improved dose distribution and shorter overall treatment course $[14,15]$. To ultimately decide upon an optimal technique for patients with seroma, we need to know which plan results in the smallest excess breast dose outside the boost planning target volume $\left(\mathrm{PTV}_{\text {boost }}\right)$. 
In this study, we compared three different boost treatment planning techniques for patients who developed seroma after BCS: a sequential boost (SEQ) based on a sequential CT scan obtained in the fifth week of RT, simultaneous integrated boost (SIB) without adaptations, and SIB adaptive radiation therapy (SIB-ART) with adaptation based on a CT scan obtained in the third week of RT. The objective was to determine whether a tighter dose distribution at the start of treatment (SIB) outweighs the dosimetric advantage of planning the boost after WBI (SEQ) for patients with seroma. Furthermore, the merit of boost re-planning during treatment (SIB-ART) was evaluated.

\section{Materials and methods \\ Patient Data}

Twenty-one patients who received breast-conserving therapy in 2008 and developed seroma in the excision cavity after undergoing microscopically complete tumor excision were consecutively selected for this study. The patients were recruited from two different RT departments within The Netherlands: The Netherlands Cancer Institute - Antoni van Leeuwenhoek Hospital, Amsterdam (n=12) and Catharina Hospital, Eindhoven $(n=9)$. No patients in our study received neo-adjuvant chemotherapy. An overview of patient characteristics is given in Table 1 .

\section{Imaging Data}

All patients underwent CT (24-slice Somatom-Sensation-Open, Siemens, Forchheim, Germany or 16slice Big-Bore, Philips Healthcare, Eindhoven, The Netherlands) as part of the standard planning for RT. Patients were scanned in supine orientation using arm and knee supports with a lead wire placed around the breast as an aid in generating the breast contour. The initial planning CT scan $\left(\mathrm{CT}_{-1}\right)$ was acquired one week prior to RT. Two additional CT scans were acquired in the third $\left(\mathrm{CT}_{3}\right)$ and fifth week $\left(\mathrm{CT}_{5}\right)$ of RT. $\mathrm{CT}_{3}$ and $\mathrm{CT}_{5}$ were registered to $\mathrm{CT}_{-1}$ using rigid registration (translations only) of bony anatomy.

\section{Planning Target Volumes and Organs at Risk}

Volumes were specifically defined and delineated for this study to avoid differences between contributing institutions. The lungs were delineated using an automatic contouring tool and visually verified. The heart was manually delineated. The total breast target volume $\left(\mathrm{TV}_{\text {breast }}\right)$ was generated based on the field borders of the radiation field. The volume was restricted to $7 \mathrm{~mm}$ inside the field borders, $7 \mathrm{~mm}$ towards the skin, $5 \mathrm{~mm}$ towards the lung, and $10 \mathrm{~mm}$ margin towards the heart. Finally, liver, stomach and ribs were removed from the volume. All patients were operated without closure of the excision cavity, therefore the post-operative seroma was defined to be the "tumor bed". The tumor bed was delineated by a trained radiation therapist using Pinnacle 8.1v (research version, Philips Radiation Oncology Systems, Milpitas, CA). All delineations were checked and approved by a radiation oncologist. To obtain the boost clinical target volume $\left(\mathrm{CTV}_{\text {boost }}\right)$, the tumor bed was expanded by $10 \mathrm{~mm}$, subsequently ribs and muscles were excluded. Finally, a PTV $_{\text {boost }}$ suitable for inverse optimization was obtained by an additional $5 \mathrm{~mm}$ expansion followed by a correction to exclude air and skin (plus $7 \mathrm{~mm}$ margin).

\section{Boost Delivery Techniques}

For each patient, three photon beams treatment plans ${ }^{1}$ were generated with Pinnacle $8.1 \mathrm{v}$ using three different planning techniques: SEQ, SIB, and SIB-ART. The linear quadratic cell survival model was used to calculate fraction sizes and total doses in SIB and SIB-ART planning [17]. The resulting SIB and SIBART plans were biologically equivalent to the SEQ plans in the total dose delivered to the target volumes in $2 \mathrm{~Gy}$ fractions assuming an $\alpha / \beta$-ratio of $10 \mathrm{~Gy}$ for the tumor bed $\left(\mathrm{BED}_{10}\right)$.

$$
S E Q \text {. }
$$

1. WBI $50.00 \mathrm{~Gy}(25 \times 2.00 \mathrm{~Gy})$ planned on $\mathrm{CT}_{-1}$,

2. Subsequently boost of $16.00 \mathrm{~Gy}(8 \times 2.00 \mathrm{~Gy})$ planned on $\mathrm{CT}_{5}$. SIB.

Total boost RT of 64.40Gy in 28 fractions including:

- WBI 50.68Gy (28x1.81Gy) planned on $\mathrm{CT}_{-1}$,

- Boost 13.72Gy (28x0.49Gy) also planned on $\mathrm{CT}_{-1}$.

\footnotetext{
${ }^{1}$ We didn't consider evaluation of electron beams for delivery of the boost dose since photon beams are preferred over electron beams especially for deep-seated targets as investigated in this study [16].
} 
SIB-ART.

After 15 fractions a new treatment plan is made based on $\mathrm{CT}_{3}$ to take part of the post-operative seroma reduction into account.

1. In the first 15 fractions boost RT of $34.50 \mathrm{~Gy}$ includes:

- WBI 27.15Gy $(15 \times 1.81 \mathrm{~Gy})$ planned on $\mathrm{CT}_{-1}$,

- Boost 7.35Gy (15x0.49Gy) planned on $\mathrm{CT}_{-1}$.

2. In the final 13 fractions boost RT of 29.90Gy includes:

- WBI 23.53Gy (13x1.81Gy) planned on $\mathrm{CT}_{3}$,

- Boost 6.37Gy (13x0.49Gy) also planned on $\mathrm{CT}_{3}$.

In terms of total dose, the administered dose in SIB-ART is the same as SIB.

Beam Setup and Plan Optimization

WBI. For all three techniques WBI treatment planning consisted of simultaneous inverse optimization of two tangential glancing rectangular fields (medial and lateral) combined with two tangential IMRT fields with identical gantry angles. The rectangular fields were positioned according to the lead wire marking the outer breast contour. In the direction of the skin a $2 \mathrm{~cm}$ margin was used to create glancing rectangular fields to take into account displacement of the breast due to breathing. Based on digitally reconstructed radiographs, Pinnacle skinviews (projection of the fields on the skin) and the CT data multileaf collimator shielding was used to protect the heart as much as possible. Optimization was limited to a maximum of eight segments $\left(\geq 9 \mathrm{~cm}^{2}\right)$ for the IMRT fields. A minimum of four monitor units were used per segment. A minimum of $80 \%$ of the whole breast dose was given with the two open fields.

Boost irradiation. One orthogonal and one or two tangential fields (depending on tumor location) were used for the boost.

For SEQ, boost fields are IMRT fields and the boost plan was created without taking into account the WBI plan. If the boundary of the PTV was $7 \mathrm{~mm}$ from the skin, the multi-leaf collimator segments were positioned around the PTV. If the PTV extended to within $7 \mathrm{~mm}$ from the skin, all leafs positioned within $7 \mathrm{~mm}$ to the skin surface were opened in the skin direction.

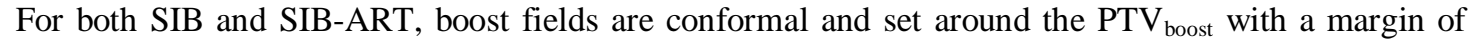
$6 \mathrm{~mm}$. Dose to boost and breast target volumes were simultaneously optimized.

Treatment planning. The setup of the tangential beams was chosen such as to achieve minimal irradiation of the contralateral breast. All plans were inversely optimized with the goal to achieve sufficient coverage of the target volumes and to minimize dose to heart and lungs. Furthermore, in order to minimize

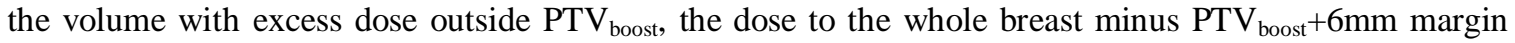
was minimized subject to the constraint that the dose to the whole breast doesn't drop below the minimal required dose. For all three techniques, at least $97 \%$ of $\mathrm{TV}_{\text {breast }}$ and $99 \%$ of $\mathrm{PTV}_{\text {boost }}$ should receive at least $95 \%$ of the prescribed dose. For all patients, plan optimization was started using a standard set of objectives. Subsequently, patient-specific tuning of the plans was performed without resetting the segments found in the initial optimization to further minimize dose to the heart and lungs.

Analysis of coverage, dose distribution and normal tissues

To establish 'treatment' doses (as opposed to 'planned' doses), for all dose distributions, a dose volume histogram $(\mathrm{DVH})$ was calculated for the volume defined by the external contour of the patient, $\mathrm{CTV}_{\text {boost }}$ and $\mathrm{PTV}_{\text {boost }}$ as defined on $\mathrm{CT}_{5}$. Target coverage was determined by evaluating the relative volumes of both $\mathrm{CTV}_{\text {boost }}$ and $\mathrm{PTV}_{\text {boost }}$ receiving $95 \%$ of the prescribed dose.

For the whole patient (including $\mathrm{PTV}_{\text {boost }}$ ), the volume that received $\geq 107 \%$ of the prescribed dose to the whole breast $\left(\mathrm{V}_{\text {patient, }} 107 \%\right.$ (breast-dose) $)$ was determined. Also the volume that received $\geq 95 \%$ of the prescribed total dose $\left(\mathrm{V}_{\text {patient, } 95 \% \text { (total-dose) }}\right)$ was determined. Furthermore, the undesired volume (outside PTV $\left._{\text {boost }}\right)$ that received $\geq 95 \%$ of the total dose $\left(\mathrm{V}_{\text {outside PTVboost, } 95 \% \text { (total-dose })}\right)[14,18]$ was determined. In addition, maximum physical dose to the absolute heart volume $\left(\mathrm{HD}_{\max }\right)$ and mean physical lung dose (MLD) were investigated.

\section{Statistical Analysis}

Since the data showed deviations from normal distributions, nonparametric statistical analyses were performed. On each of the variables obtained from the dose distributions a Friedman test was performed to establish differences between planning techniques. All significant effects were investigated post-hoc using Wilcoxon-signed-ranks tests. The significance of seroma reduction over time was determined by one- 
sample t test. Furthermore, for each pair of treatment plans, we determined the correlations between the difference in $\mathrm{V}_{\text {outside PTVboost, 95\%(total-dose) }}$ on the one hand and seroma volume as well as seroma volume reduction on the other hand. Correlations were computed as the Spearmen ranked correlation. Statistical significance was assumed at $p \leq 0.05$.

\section{Results}

All generated treatment plans adhered to clinical requirements. An overview of the target coverage is

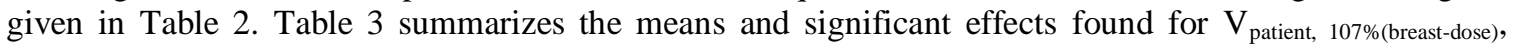
$\mathrm{V}_{\text {patient, 95\%(total-dose) }}, \mathrm{V}_{\text {outside PTVboost, 95\%(total-dose) }}, \mathrm{HD}_{\max }$ and MLD.

\section{Coverage and Dose Distribution}

All three techniques provide similar $\mathrm{CTV}_{\text {boost }}$ coverage. SIB and SIB-ART perform slightly worse compared to SEQ concerning PTV $\mathrm{V}_{\text {boost }}$ coverage.

Figure 1 illustrates the total dose distribution $\left(\mathrm{V}_{\text {patient, } 107 \% \text { (breast-dose), }}, \mathrm{V}_{\text {patient, } 95 \% \text { (total-dose) }}\right)$ on $\mathrm{CT}_{5}$ together with the tumor bed and $\mathrm{PTV}_{\text {boost }}$ for the three different techniques for one patient. $\mathrm{V}_{\text {patient, } 107 \% \text { (breast-dose) }}$ was smallest for SIB-ART, followed by SIB and then SEQ $(p<0.001)$. $\mathrm{V}_{\text {patient, } 107 \% \text { (breast-dose) }}$ was on average reduced by $16 \%$ and $9 \%$ with SIB-ART when compared to SEQ and SIB, respectively, and a $7 \%$ reduction was demonstrated with SIB when compared to SEQ.

$\mathrm{V}_{\text {patient, } 95 \% \text { (total-dose) }}$ was on average reduced by $13 \%$ and $19 \%(p<0.001)$ with SIB-ART when compared to SEQ and SIB, respectively. Also a statistically significant difference in $\mathrm{V}_{\text {patient, }}$ 95\%(total-dose) was found between SEQ and SIB ( $p=0.003)$ with an average increase of $8 \%$ with SIB.

\section{Normal Tissues}

The difference in physical $\mathrm{HD}_{\max }$ for patients with left-sided breast cancer ( $\left.\mathrm{n}=11\right)$ was statistically significant between the three techniques $(p \leq 0.04)$. SIB-ART demonstrated the smallest mean volume, followed by SIB and then SEQ. For patients with right-sided breast cancer $(n=10)$, the smallest mean $\mathrm{HD}_{\max }$ was found with SEQ. The difference in physical $\mathrm{HD}_{\max }$ was statistically significant when comparing SEQ to both SIB and SIB-ART ( $p=0.03$ and $p=0.01$ respectively). No significant difference was found between SIB and SIB-ART.

The difference in physical MLD between the three techniques was small but statistically significant $(p \leq 0.02)$. The smallest mean MLD was found with SEQ followed by SIB-ART and SIB.

The difference in $\mathrm{V}_{\text {outside }}$ PTVboost, 95\%(total-dose) was found to be statistically significant for SIB-ART compared to both SEQ and SIB $(p<0.001)$. On average a reduction of $35 \%$ and $26 \%$ was observed with SIB-ART when compared to SIB and SEQ, respectively.

\section{Seroma Volume, Seroma Reduction and Excess Dose}

The mean seroma volumes for $\mathrm{CT}_{-1}, \mathrm{CT}_{3}$ and $\mathrm{CT}_{5}$ were $63 \mathrm{~cm}^{3}$ (range: $18 \sim 218 \mathrm{~cm}^{3}$ ), $32 \mathrm{~cm}^{3}$ (range: $\left.8 \sim 122 \mathrm{~cm}^{3}\right)$ and $25 \mathrm{~cm}^{3}\left(\right.$ range: $\left.6 \sim 102 \mathrm{~cm}^{3}\right)$, respectively. Significant total seroma reduction during RT was demonstrated (mean $=62 \%$, range: $38 \sim 85 \%, p<0.001$, one-sample t-test). Larger seroma reduction (77\% of the total reduction) was observed between $\mathrm{CT}_{-1}$ and $\mathrm{CT}_{3}$ than between $\mathrm{CT}_{3}$ and $\mathrm{CT}_{5}(p=0.001$, Wilcoxonsigned-ranks test).

The absolute difference in $\mathrm{V}_{\text {outside PTVboost, } 95 \% \text { (total-dose) }}$ between SIB and SIB-ART was found to be statistically significantly correlated with both seroma volume on $\mathrm{CT}_{-1}\left(p=0.002, \mathrm{r}_{\mathrm{s}}=0.6\right)$ and absolute seroma reduction between $\mathrm{CT}_{-1}$ and $\mathrm{CT}_{3}\left(p=0.001, r_{\mathrm{s}}=0.7\right)$ (Fig. 2). This indicates that patients with a larger initial seroma volume or a larger seroma reduction between $\mathrm{CT}_{-1}$ and $\mathrm{CT}_{3}$ benefit more from SIB-ART compared to SIB.

\section{Discussion}

The aim of this study was to retrospectively compare three boost RT planning techniques (SEQ, SIB and SIB-ART) in patients who developed seroma after breast-conserving surgery. This study showed that irradiated boost volumes were significantly smaller with SIB-ART, despite the apparent advantage of a sequential boost with respect to seroma shrinkage. The three techniques only slightly differed in dose delivery to the lungs and heart. Therefore, our study indicates a clinical preference for SIB-ART in postoperative breast cancer patients with seroma. 


\section{Comparing SEQ, SIB and SIB-ART}

Although SEQ, SIB and SIB-ART were planned according to their clinical protocol, performance evaluation was done on a CT scan acquired in the fifth week of $\mathrm{RT}\left(\mathrm{CT}_{5}\right)$. This ensured a clinical comparison, rather than a planning comparison, accounting for and comparing the techniques including the features inherent to SEQ and SIB(-ART). On the one hand, since boost planning for SEQ is actually performed on $\mathrm{CT}_{5}$, and our patients demonstrate significant seroma reduction during RT, this provided an advantage for SEQ planning when compared to SIB(-ART). On the other hand, the combination of the slightly lower physical dose (to obtain biologically equivalent plans) with inherently tighter total planned dose distributions $[14,18]$ provided an advantage for $\operatorname{SIB}(-\mathrm{ART})$. In this study, these features were deliberately maintained to provide a fair clinical comparison.

\section{Coverage and Dose Distribution}

We found that overall irradiated volumes (whole breast as well as boost) were always significantly smaller with SIB-ART compared to both SIB and SEQ. Nevertheless, boost target coverage was slightly worse for both SIB and SIB-ART compared to SEQ. This is due to geometrical changes in breast tissue that occurred in three patients during the third and fifth week of RT. Therefore, boost target volumes assessed for SIB and SIB-ART in CT scans obtained pre-treatment and in the third week of RT were no longer representative of the boost volumes in the fifth week of RT for these patients. This finding indicates the importance of monitoring the excision cavity for changes during RT in order to re-define target volume and modify treatment plans when needed [19].

\section{Normal Tissues}

For patients with right-sided breast cancer, $\mathrm{HD}_{\max }$ was found to be significantly lower for SEQ when compared to both SIB and SIB-ART. Nevertheless, since RT for patients with right-sided breast cancer generally involves smaller cardiac dose delivery, $\mathrm{HD}_{\max }$ was for all three techniques smaller than $8 \mathrm{~Gy}$ on average. It is therefore to be questioned whether the small differences in dosage have any clinical impact [20].

For all three techniques, the average mean physical lung dose was well below the maximum normalized total lung dose $(7.5 \mathrm{~Gy})$ that could result in less than $1 \%$ risk of radiation pneumonitis Grade 2 or higher [21].

Since boost treatment planning for SEQ is planned on a CT scan obtained in the fifth week of RT, one might expect superiority of the performance of SEQ when evaluating $V_{\text {outside PTVboost, } 95 \% \text { (total-dose) }}$ in the fifth week of RT. Conversely, evaluation of the performance of the three techniques in this study showed a reduction of $26 \%$ in $\mathrm{V}_{\text {outside }}$ PTVboost, $95 \%$ (total-dose) when using SIB-ART compared to SEQ. On the other hand, an increase of $16 \%$ in $\mathrm{V}_{\text {outside }}$ PTVboost, 95\%(total-dose) was observed when using SIB compared to SEQ. Note that the performances of SIB and SIB-ART are biased to their disadvantage as a consequence of the evaluation of the total plan on $\mathrm{CT}_{5}$. Nevertheless, the current results indicate a preference for SIB-ART with respect to minimizing $\mathrm{V}_{\text {outside PTVboost, } 95 \% \text { (total-dose). }}$

\section{Clinical Implications}

Our study showed that for patients who demonstrated seroma reduction, a SIB-ART planning technique is clinically preferable to a SEQ planning technique as the combined effect of a tighter dose distribution and re-planning midway through RT is most successful in achieving excess dose reduction. The clinical implication would be replacing SEQ with SIB(-ART). Nevertheless, a difficult issue remains: what specific dosimetric gain is clinically relevant enough to spend the additional time associated with re-planning? For each $100 \mathrm{~cm}^{3}$ increase in irradiated boost volume a fourfold increase in risk of fibrosis was observed [10]. Therefore, a reduction of $50 \mathrm{~cm}^{3}$ in volume of excess dose during RT can be considered clinically significant and should be taken into consideration. The graphs in Figure 2 could be used to derive clinical indications for the decision to start monitoring and to create the adaptive plan. Two thresholds can be derived: 1) patients with an initial seroma volume $\geq 40 \mathrm{~cm}^{3}$ should be monitored and 2) patients with seroma reduction of $\geq 20 \mathrm{~cm}^{3}$ in the first four weeks after acquisition of the initial planning CT should be re-planned. In our investigated patient group $48 \%$ of the patients would benefit (reduction of $\geq 50 \mathrm{~cm}^{3}$ in volume of excess dose) from SIB-ART compared to SIB. However, whether these thresholds are effective should be addressed in a larger patient group in future investigations.

Variation in boost target volume delineation in breast RT is a well known issue [22-24]. However, it has been shown that changes in boost volume during RT due to seroma shrinkage are significant in relation to 
clinical interobserver variations [25]. Furthermore, delineation variation is smaller when seroma is present [22,24]. In case SIB-ART is used in clinical practice it is advisable to have all delineation for a specific patient done by the same physician. This way delineation variation is minimized and undesirable adaptation of the location of the boost target volume is prevented.

\section{Limitations}

It is to be questioned whether SIB-ART would be preferable to SIB in a subselection of patients that demonstrates minor seroma reduction in the first three weeks of RT. This was not investigated in our current study and should be addressed in future investigations.

The clinical fractionation schedule for SIB was calculated using an $\alpha / \beta$-ratio of $10 \mathrm{~Gy}$. However, recent data suggest lower $\alpha / \beta$-ratio (e.g. 3.6Gy) could be more applicable [26,27]. Nevertheless, our study represents the current clinical practice in terms of breast RT dosing.

\section{Conclusions}

Seroma is frequently seen in patients after breast-conserving surgery. Reduction is normally seen during the first weeks of adjuvant radiotherapy [3,9]. Our study demonstrates that, for patients with seroma reduction, a tighter dose distribution from the start of treatment (SIB) outweighs the advantage of sequential boost planning (SEQ) provided that the delivery of SIB is re-planned halfway through treatment. This leads us to the conclusion that SIB-ART is the optimal boost radiation treatment planning technique and should be considered for standard clinical practice.

\section{Conflict of interest statement}

The authors of this manuscript have no actual or potential conflicts of interest to disclose.

\section{Acknowledgments}

The authors would like to thank J. van der Leer, RT(T) (Department of Radiation Therapy, Catharina Hospital, Eindhoven, The Netherlands) and D. Minkema, RT(T) (Department of Radiation Oncology, The Netherlands Cancer Institute - Antoni van Leeuwenhoek Hospital, Amsterdam, The Netherlands) for their contributions to this study. Financial support of this work was provided by the Dutch Cancer Society (Grant No. KWF 2008-4024).

\section{References}

1. Clarke M, Collins R, Darby S, et al. Effects of radiotherapy and of differences in the extent of surgery for early breast cancer on local recurrence and 15-year survival: an overview of the randomised trials. Lancet 2005;366(9503):2087-2106.

2. Bartelink H, Horiot JC, Poortmans PM, et al. Impact of higher radiation dose on local control and survival in breast-conserving therapy of early breast cancer: 10-year results of the randomized boost versus no boost EORTC 22881-10882 trial. J Clin Oncol 2007;25(22):3259-3265.

3. Kader HA, Truong PT, Pai R, et al. When is CT-based postoperative seroma most useful to plan partial breast radiotherapy? Evaluation of clinical factors affecting seroma volume and clarity. Int $\mathrm{J}$ Radiat Oncol Biol Phys 2008;72(4):1064-1069.

4. Prendergast B, Indelicato DJ, Grobmyer SR, et al. The dynamic tumor bed: volumetric changes in the lumpectomy cavity during breast-conserving therapy. Int J Radiat Oncol Biol Phys 2009;74(3):695-701.

5. Hurkmans CW, Borger JH, Pieters BR, et al. Variability in target volume delineation on CT scans of the breast. Int J Radiat Oncol Biol Phys 2001;50(5):1366-1372.

6. Jacobson G, Betts V, Smith B. Change in volume of lumpectomy cavity during external-beam irradiation of the intact breast. Int J Radiat Oncol Biol Phys 2006;65(4):1161-1164.

7. Oh KS, Kong FM, Griffith KA, et al. Planning the breast tumor bed boost: changes in the excision cavity volume and surgical scar location after breast-conserving surgery and whole-breast irradiation. Int $\mathbf{J}$ Radiat Oncol Biol Phys 2006;66(3):680-686.

8. Tersteeg RJ, Roesink JM, Albregts M, et al. Changes in excision cavity volume: prediction of the reduction in absolute volume during breast irradiation. Int J Radiat Oncol Biol Phys 2009;74(4):11811185.

9. Yang TJ, Elkhuizen PH, Minkema D, et al. Clinical factors associated with seroma volume reduction in breast-conserving therapy for early stage breast cancer, a multi-institutional analysis. Int J Radiat Oncol Biol Phys 2010;76(5):1325-1332. 
10. Borger JH, Kemperman H, Smitt HS, et al. Dose and volume effects on fibrosis after breast conservation therapy. Int J Radiat Oncol Biol Phys 1994;30(5):1073-1081.

11. Collette S, Collette L, Budiharto T, et al. Predictors of the risk of fibrosis at 10 years after breast conserving therapy for early breast cancer - A study based on the EORTC trial 22881-10882 'boost versus no boost'. Eur J Cancer 2008;44(17):2587-2599.

12. Hijal T, Fournier-Bidoz N, Castro-Pena P, et al. Simultaneous integrated boost in breast conserving treatment of breast cancer: a dosimetric comparison of helical tomotherapy and three-dimensional conformal radiotherapy. Radiother Oncol 2010;94(3):300-306.

13. Van der Laan HP, Dolsma WV, Schilstra C, et al. Limited benefit of inversely optimised intensity modulation in breast conserving radiotherapy with simultaneously integrated boost. Radiother Oncol 2010;94(3):307-312.

14. Hurkmans CW, Meijer GJ, van Vliet-Vroegindeweij C, et al. High-dose simultaneously integrated breast boost using intensity-modulated radiotherapy and inverse optimization. Int $\mathrm{J}$ Radiat Oncol Biol Phys. 2006;66(3):923-930.

15. McDonald MW, Godette KD, Whitaker DJ, et al. Three-year outcomes of breast intensity-modulated radiation therapy with simultaneous integrated boost. Int J Radiat Oncol Biol Phys 2010;77(2):523-530.

16. Toscas JI, Linero D, Rubio I, et al. Boosting the tumor bed from deep-seated tumors in early-stage breast cancer: a planning study between electron, photon, and proton beams. Radiother Oncol 2010;96(2):192-198.

17. Joiner MC, van der Kogel AJ. The linear-quadratic approach to fractionation and calculation of isoeffect relationships. In: Steel GG, editor. Basic clinical radiobiology. $2^{\text {nd }}$ ed. London: Arnold; 1997. p. 106-122.

18. van der Laan HP, Dolsma WV, Maduro JH, et al. Three-dimensional conformal simultaneously integrated boost technique for breast-conserving radiotherapy. Int $\mathrm{J}$ Radiat Oncol Biol Phys 2007;68(4):1018-1023.

19. Yang TJ, Minkema D, Elkhuizen PH, et al. Clinical applicability of cone-beam CT in monitoring seroma volume change during breast irradiation. Int J Radiat Oncol Biol Phys 2010;78(1):119-126.

20. Taylor CW, Povall JM, McGale P, et al. Cardiac dose from tangential breast cancer radiotherapy in the year 2006. Int J Radiat Oncol Biol Phys 2008;72(2):501-507.

21. Kwa SLS, Lebesque JV, Theuws JCM, et al. Radiation pneumonitis as a function of mean lung dose: an analysis of pooled data of 540 patients. Int J Radiat Oncol Biol Phys 1998;42(1):1-9.

22. Petersen RP, Truong PT, Kader HA, et al. Target volume delineation for partial breast radiotherapy planning: clinical characteristics associated with low interobserver concordance. Int J Radiat Oncol Biol Phys 2007;69:41-48.

23. Struikmans H, Wárlám-Rodenhuis C, Stam T, et al. Interobserver variability of clinical target volume delineation of glandular breast tissue and of boost volume in tangential breast irradiation. Radiother Oncol 2005;76:293-299.

24. van Mourik AM, Elkhuizen PH, Minkema D, et al. Multiinstitutional study on target volume delineation variation in breast radiotherapy in the presence of guidelines. Radiother Oncol 2010;94(3):286-291.

25. Hurkmans C, Admiraal M, van der Sangen M, et al. Significance or breast boost volume changes during radiotherapy in relation to current clinical interobserver variations. Radiother Oncol 2009;90(1):60-65.

26. Owen JR, Ashton A, Bliss JM, et al. Effect of radiotherapy fraction size on tumour control in patients with early-stage breast cancer after local tumour excision: long-term results of a randomised trial. Lancet Oncol 2006;7(6):467-471.

27. Qi XS, White J, Li XA. Is $\alpha / \beta$ for breast cancer really low? Radiother Oncol 2011. Article In Press. 


\section{Figures}

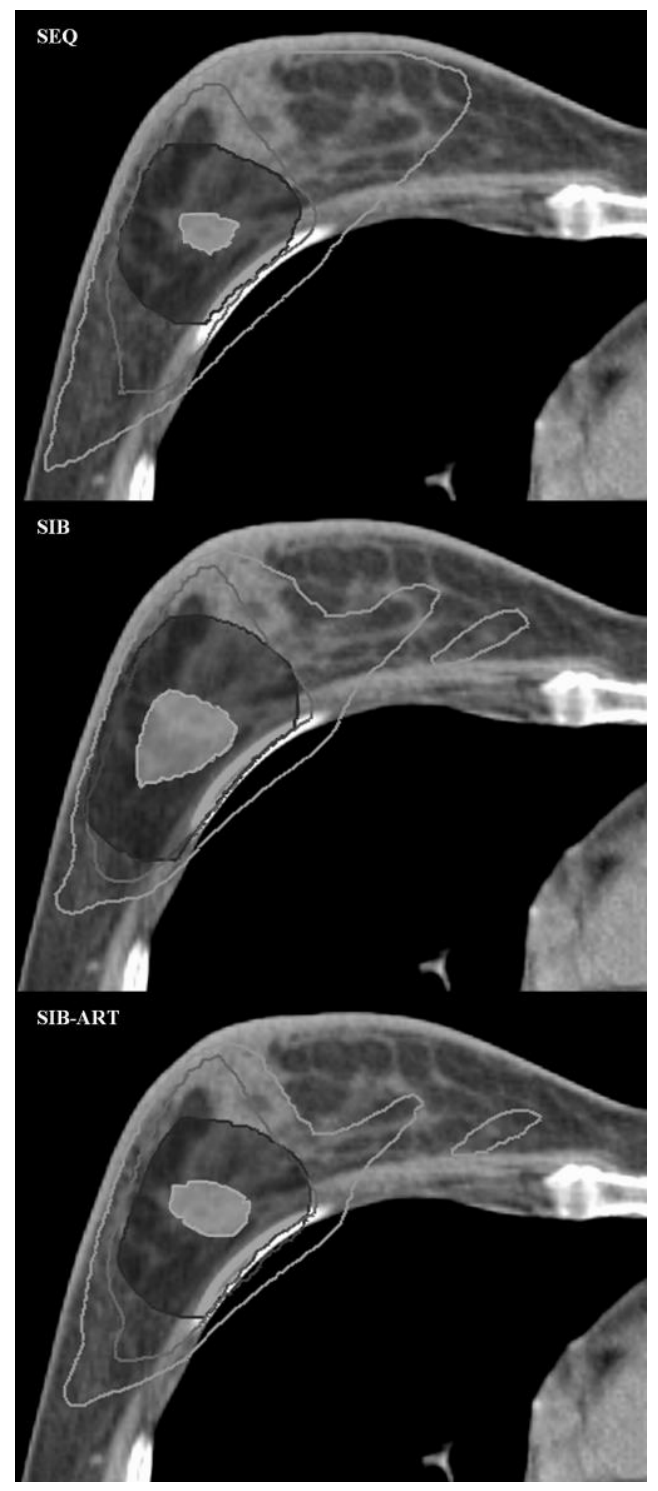

Figure 1 An illustration of the total dose distribution on the CT scan acquired in the fifth week of RT. Both the volume that received $\geq 107 \%$ of the prescribed dose to the whole breast (light grey line) and the volume that received $\geq 95 \%$ of the prescribed total dose (dark grey line) are illustrated. Also the tumor bed (white overlay) and boost planning target volume (black overlay) used for planning are visualized. The tumor bed and boost planning target volume visualized are derived from the initial planning CT scan acquired before RT, CT scan acquired in the third and fifth week of RT for SIB, SIB-ART and SEQ respectively. 

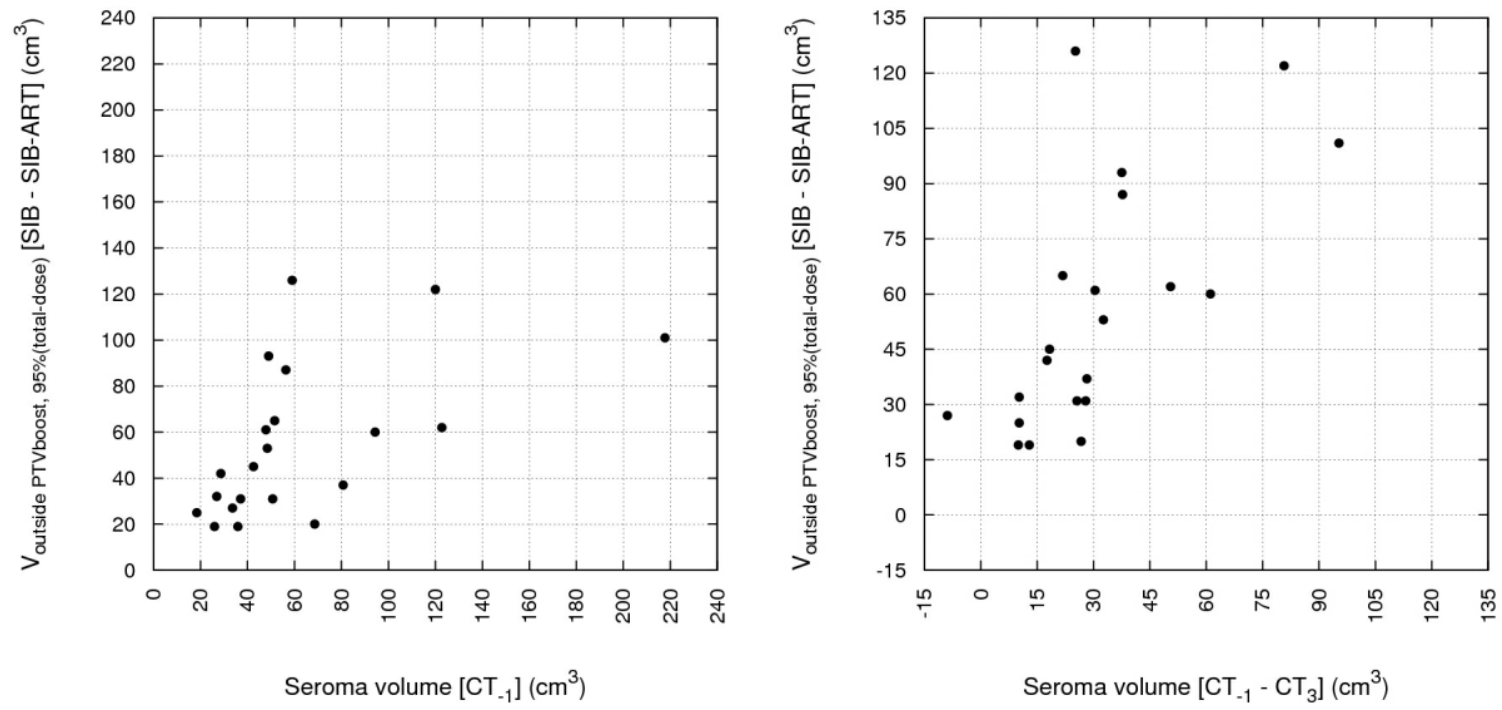

Figure 2 Left: Seroma volume derived from the initial planning CT scan $\left(\mathrm{CT}_{-1}\right)$ versus the difference between SIB and SIB-ART in undesired volume receiving $\geq 95 \%$ of the total dose ( $\mathrm{V}_{\text {outside PTVboost, } 95 \% \text { (total- }}$ dose) ) evaluated in the fifth week of RT. Right: Seroma reduction between $\mathrm{CT}_{-1}$ and the $\mathrm{CT}$ scan acquired in the third week of RT $\left(\mathrm{CT}_{3}\right)$ versus the difference in $\mathrm{V}_{\text {outside PTVboost, } 95 \% \text { (total-dose) }}$ evaluated in the fifth week of RT. 
Alderliesten 10

Tables

Table 1. Patient characteristics.

\begin{tabular}{|c|c|c|}
\hline Patients (n) & & 21 \\
\hline Age (y) at initiation of radiation therapy & Mean (Range) & $59(38 \sim 81)$ \\
\hline T stage (n) & $\begin{array}{l}\text { Tis } \\
\text { T1 } \\
\text { T2 }\end{array}$ & $\begin{array}{c}1 \\
16 \\
4\end{array}$ \\
\hline $\mathrm{N}$ stage $(\mathrm{n})$ & $\begin{array}{l}\text { N0 } \\
\text { N1 } \\
\text { N3 }\end{array}$ & $\begin{array}{c}17 \\
3 \\
1\end{array}$ \\
\hline M stage (n) & M0 & 21 \\
\hline Time between surgery and $\mathrm{CT}_{-1}$ (days) & Mean (Range) & $27(15 \sim 48)$ \\
\hline Time between surgery and $\mathrm{CT}_{3}$ (days) & Mean (Range) & $51(35 \sim 75)$ \\
\hline Time between surgery and $\mathrm{CT}_{5}$ (days) & Mean( Range) & $68(52 \sim 89)$ \\
\hline Actual radiation treatment $(\mathrm{n})$ & $\begin{array}{c}\text { SEQ } \\
\text { SIB } \\
\text { SIB-ART }\end{array}$ & $\begin{array}{c}4 \\
11 \\
6\end{array}$ \\
\hline
\end{tabular}

\section{Abbreviations:}

$\mathrm{CT}_{-1}$ : initial planning $\mathrm{CT}$ acquired before start RT.

$\mathrm{CT}_{3}$ : CT scan acquired in the third week of RT.

$\mathrm{CT}_{5}$ : CT scan acquired in the fifth week of RT.

Table 2. Target coverage (relative volumes receiving 95\% of the prescribed dose) results are presented as mean values with ranges in parentheses. The coverage for $\mathrm{PTV}_{\text {boost }}$ and $\mathrm{CTV}_{\text {boost }}$ defined on the $\mathrm{CT}$ scan acquired in the fifth week of RT are given.

\begin{tabular}{lccc}
\hline Variable & SEQ & SIB & SIB-ART \\
\hline PTV $_{\text {boost }}$ & $100.0(99.6 \sim 100.0)$ & $98.7(91.7 \sim 100.0)$ & $97.6(83.0 \sim 100.0)$ \\
CTV $_{\text {boost }}$ & $99.7(98.0 \sim 100.0)^{*}$ & $99.8(99.0 \sim 100.0)$ & $99.6(94.8 \sim 100.0)$ \\
\hline
\end{tabular}

*Under-dosage within $C T V_{\text {boost }}\left(\right.$ e.g. tumor bed near the skin) has a larger effect on $C T V_{\text {boost }}$ coverage than on PTV $V_{\text {boost }}$ coverage when expressing the coverage as a percentage of the volume. 
Table 3. Means of all investigated variables for the three different planning techniques, $p$-values of Friedman tests are reported as well as an indication of the specific effect as found by Wilcoxon-signedranks tests. For example, SIB-ART $<$ SIB $<$ SEQ means that all differences are significant. SEQ $\sim$ SIB $\sim$ SIBART means that none of the differences are significant.

\begin{tabular}{|c|c|c|c|c|c|c|}
\hline \multirow{2}{*}{\multicolumn{2}{|c|}{ Variable }} & \multicolumn{3}{|c|}{ mean } & \multirow{2}{*}{$\begin{array}{c}\text { Friedman } \\
p\end{array}$} & \multirow[t]{2}{*}{ Wilcoxon } \\
\hline & & SEQ & SIB & SIB-ART & & \\
\hline \multicolumn{2}{|c|}{$\mathrm{V}_{\text {patient, } 107 \% \text { (breast-dose) }}\left(\mathrm{cm}^{3}\right)$} & 584.5 & 536.8 & 485.5 & $<0.001$ & SIB-ART $<$ SIB $<$ SEQ \\
\hline \multicolumn{2}{|c|}{$\mathrm{V}_{\text {patient, } 95 \%(\text { total-dose) }}\left(\mathrm{cm}^{3}\right)$} & 273.6 & 289.4 & 234.2 & $<0.001$ & SIB-ART $<$ SEQ $<$ SIB \\
\hline \multicolumn{2}{|c|}{$\mathrm{V}_{\text {outside }}$ PTVboost, $95 \%$ (total-dose) $\left(\mathrm{cm}^{3}\right)$} & 134.4 & 150.1 & 95.0 & $<0.001$ & SIB-ART $<$ SEQ $<$ SIB \\
\hline $\mathrm{HD}_{\max } *(\mathrm{~Gy})$ & $\begin{array}{l}\text { Left } \\
\text { Right }\end{array}$ & $\begin{array}{c}39.9 \\
5.7\end{array}$ & $\begin{array}{c}36.9 \\
7.4\end{array}$ & $\begin{array}{c}35.8 \\
7.3\end{array}$ & $\begin{array}{c}0.001 \\
0.03\end{array}$ & $\begin{array}{l}\text { SIB }-A R T<\text { SIB }<\text { SEQ } \\
\text { SEQ }<\text { SIB }, \text { SEQ }<\text { SIB-ART, SIB } \sim \text { SIB-ART }\end{array}$ \\
\hline MLD* (Gy) & & 4.2 & 4.6 & 4.5 & 0.001 & $\mathrm{SEQ}<\mathrm{SIB}-\mathrm{ART}<\mathrm{SIB}$ \\
\hline
\end{tabular}

Abbreviations:

$\mathrm{V}_{\text {patient, } 107 \% \text { (breast-dose): }}$ volume of the patient that received $\geq 107 \%$ of the prescribed dose to the whole breast.

$\mathrm{V}_{\text {patient, } 95 \% \text { (total-dose) }}$ : volume of the patient that received $\geq 95 \%$ of the prescribed total dose.

$\mathrm{V}_{\text {outside PTVboost, } 95 \% \text { (total-dose) }}$ : undesired volume receiving $\geq 95 \%$ of the total dose.

$\mathrm{HD}_{\max }$ : maximum physical dose to the absolute heart volume.

MLD: mean physical lung dose.

*: Derived from dose distributions that are uncorrected for fractionation effects.

Left: left-sided breast cancer.

Right: right-sided breast cancer. 